\title{
Soret and Dufour Effects on Heat and Mass Transfer Mixed Convection Flow near a Point of Zero Skin Friction
}

\author{
Ashraf $\mathrm{M}^{*}$, Mahmood T, Ahmad $\mathrm{U}$ and Hassan W \\ Department of Mathematics, Faculty of Science, University of Sargodha, Sargodha, Pakistan
}

\begin{abstract}
Present study concerns with the computational analysis of boundary-layer mixed convection flow in the vicinity of separation along vertical flat plate in the presence of Soret and Dufor effects. Properties of the fluid are considered to be viscous, incompressible, steady and two dimensional. The formulation of the nonlinear coupled partial differential equations is based on the properties and characteristics of the fluid and fluid flow domain. Formulated partial differential equations for the flow model are transformed into convenient form by using some algebraic manipulation to nd the numerical solution of the proposed model. The main purpose of this study is to analyze the Soret and Dufor effects on chief physical quantities that is on skin friction, rate of heat transfer, mass flux, mass concentration, momentum and thermal boundary layer thicknesses. From the result reports in this study, it can be seen that velocity profile increase while the temperature distribution and mass concentration is decreased with the increase of mixed convection parameter, $\lambda_{T}$ and opposite behavior is noted for modified mixed convection parameter, $\lambda_{c}$. Further, it is also shown that skin friction, rate of heat transfer and mass transfer are increased for various values of mixed convection parameter, $\lambda_{t}$ as well as Prandtl numeber Pr. Present obtained results are compared with the results given in literature and found be in good agreement.
\end{abstract}

Keywords: Mixed convection; Flat plate; Dufour effects; Soret effects

\section{Nomenclature}

$C_{1}$ : Mass concentration at the Surface; $C_{0}$ : Concentration in Free Stream; $c_{p}$ : Specific Heat at Constant Pressure; $C_{s}$ : Concentration Susceptibility; $D a$ : Darcy Number; $D_{B}$ : Brownion Difussion; $D_{m}$ : Mass Difussivity; Du: Dufour Number; Gr: Grash of Number; G: Gravitational Acceleration, $\left[\mathrm{ms}^{-2}\right]$; K: Thermal Conductance of the Fluid; $K_{T}$ : Thermal Diffusion Ratio; l: Characteristics Length ; Pr: Prandtl Number; Q: Rate of Heat Transfer; Q: Rate of Mass Transfer; Re: Reynolds Number; $S c$ : Schmidth Number; $T_{1}$ : Temperature at the Surface; $T_{0}$ : Free Stream Temperature; $U$ : Free Stream Velocity; $U$ : Velocity along X-Axis; $V$ : Velocity along Y-Axis.

Greek letters: $\theta$ : Dimensionless Temperature Function; $\Psi$ : Fluid Stream Function, $\left[\mathrm{m}^{2} \mathrm{~s}^{-1}\right] ; \eta$ : Similarity Transformation: $v$ : Kinematic Viscosity, $\left[\mathrm{m}^{2} \mathrm{~s}^{-1}\right]$; Fi: Coefficient of Thermal Expansion; $\varphi$ : Transformed Stream Function; $\lambda_{\mathrm{T}}$ : Mixed Convection Parameter; $\lambda_{\mathrm{C}}$ : Modified Mixed Convection Parameter; $\tau_{w}$ : Shear Stress;

Subscripts: 1: Surface Condition; 0: Ambient Condition.

\section{Introduction}

The relation between heat and mass transfer behaviour by mixed convection flow receives in heating and cool-ing processes in semiconductor electronics, solar energy systems, transport phenomena in power transformer electronics, absorption reactors, binary diffusion systems and polymer processing in the plastic industry etc. When both heat and mass transfer exists simultaneously between the fluxes, the fluid nature become more complex because energy flux not only generates by temperature gradients but also by concentration gradients. The energy flux created by mass concentration gradient is called Dufour (diffusion thermo) effects. It is the reciprocal phenomena of Soret (thermal diffusion) effects which is created by temperature gradients. With this understanding we highlight the work by scientists and researchers, they did in the past.

Hunt and Wilks [1] investigated a point of zero skin friction on behavior of the laminar boundary layer mixed convection flow. Kafoussias and Williams [2] have studied the mixed convection heat and mass transfer boundary layer flow when the viscosity is varied with the temperature. Postelnicu [3] carried out Soret and Dufour effects with inluence of a magnetic field on heat and mass transfer by natural convection from vertical surface in porous media. He observed that thickness of hydrodynamic boundary layer increases as magnetic parameter increases. Abreu et al. [4] have developed the physical model of forced and natural convection boundary layer flow in the presence of Soret and Dufour effects and analyzed that the range of velocity field increases for a negative value of Dufour and Soret coefficient and decreases for positive values. Chamkha and Nakhi [5] investigated Soret and Dufour effects on magnetohydrodynamics mixed convection radiation interaction along a permeable surface immersed in a porous medium. They predicted that in the presence of suction, the local Nusselt number decreases with the increase in Dufour number. Beg et al. [6] used local non- similarity method to obtain Soret and Dufour effects on chemically-reacting mixed convec-tion heat and mass transfer along inclined and vertical plates. Mixed convection heat and mass transfer in porous medium with Soret and Dufour effects was studied by Slam [7]. Makinde et al. [8] highlighted Soret and Dufour effects past a vertical plate embedded in a porous medium with magnetohydrodynamics mixed convection flow. They concluded that local skin friction on the surface of the plate increases by increasing the values of Eckert number, Soret and Dufour numbers. Pal and Mondal [9] used Runge Kutta Fehlberg integration scheme to consider chemical reaction and

*Corresponding author: Ashraf M, Department of Mathematics, Faculty of Science, University of Sargodha, Sargodha, Pakistan, Tel: 92-48-9230811-15 E-mail: ashrafcfd@uos.edu.pk

Received March 21, 2018; Accepted July 02, 2018; Published July 10, 2018

Citation: Ashraf M, Mahmood T, Ahmad U, Hassan W (2018) Soret and Dufour Effects on Heat and Mass Transfer Mixed Convection Flow near a Point of Zero Skin Friction. Fluid Mech Open Acc 5: 184. doi: 10.4172/2476-2296.1000184

Copyright: (C) 2018 Ashraf M, et al. This is an open-access article distributed under the terms of the Creative Commons Attribution License, which permits unrestricted use, distribution, and reproduction in any medium, provided the original author and source are credited. 
thermal radiation for magnetohydrodynamics non-Darcy unsteady mixed convective heat and mass transfer over a stretching sheet with Soret and Dufour effects and found that Soret number leads to increase skin friction coefficient and Nusselt number. Gundagani et al. [10] worked for unsteady magnetohydrodynamics mixed convection flow past a vertical porous plate with thermal radiation, Soret and Dufour effects and found the inluence of di erent value of parameters on the velocity, temperature and concentration profiles. The mathematical model of Soret and Dufour effects on unsteady heat and mass transfer magnetohydrodynamics mixed convection flow over an impulsively stretched vertical surface with chemical reaction was investigated by Chamakha and Kabeir [11]. They obtained that skin friction coefficient and local Nusselt number decreases as Dufour number increases. The problem on Magnetohydrodynamics mixed convection heat and mass transfer in a micro polar fluid with Soret and Dufour effects was solved by Srinivasachary and Upendar [12], where they found that Nusselt number increases as magnetic parameter is increased. Chamkha and Rashad [13] discussed Soret and Dufour effects on un-steady heat and mass transfer magnetohydrodynamics mixed convection flow from a rotating vertical cone with chemical reaction. They described the effect of buoyancy, chemical and magnetic parameter on the local tangential and azimuthal skin friction coefficient. Muthuraj et al. [14] studied magnetohydrodynamics mixed convection flow of micropolar fluid in a vertical channel with viscous dissipation, Soret, Dufour and space porosity effects. They used Homotopy analysis method to obtain the approximate analytical solution for the velocity, micro-rotation, temperature, and concentration field. Khidir and Sibanda [15] have con-sidered magnetohydrodynamics mixed convective flow from an exponentially stretching surface in porous media with cross-diffusion and effects of temperature dependent viscosity. They analyzed the behavior of skin friction, heat and mass transfer for various values of physical parameters. Arthur et al. [16] elaborated hydromagnetics flow past a vertical plate embedded in a porous medium, they claimed that embedded pa-rameters can control kinematics, heat and mass transfer process. Srinivasacharys et al. [17] studied Soret and Dufour effects on mixed convection flow along a vertical wavy surface in a porous medium with variable fluid properties. They solved the model numerically and reported the results of velocity, temperature and mass concentration as well as Nusselt and Sherwood numbers graphically. $\mathrm{Pal}$ et al. [18] have examined magnetohydrodynamics convectiveradiative heat and mass transfer of nanofluids over a vertical nonlinear stretching/shrinking sheet with Soret and Dufour effects and investigated that Skin-friction coefficient de-creases for stretching sheet but opposite effect is found for shrinking sheet by decreasing the value of Soret number and increasing the values of Dufour number. Soret and Dufour effects on unsteady magnetohydro-dynamics mixed convection flow of chemically and radiating couple stress fluid in a porous medium between parallel plates was investigated by Ojjeela and Kumar [19] and observed that the temperature of the fluid enhanced, whereas the concentration reduced with the increase in Soret and Dufour parameters. Abel et al. [20] have discussed analytical solution of mixed convection heat transfer of magnetohydrodynamics flow due to permeable sheet and investigated that thermal boundary layer thickness increases with an increase in the value of Dufour number. In keeping view above literature survey we formulate a mathematical model of mixed convection flow near a point of zero skin friction with Soret and Dufour effects.

\section{Mathematical Model and Governing Equations}

Consider steady state two dimensional laminar boundary layer flow of viscous incompressible fluid along a semi-infinite flat plate with uniform temperature $T_{1}$ and ambient temperature $T_{0}$ extending vertically with its leading edge. The $x$-axis is taken along the plate and $y$-axis is normal to it. The dimensioned boundary layer equations by following Hunt and Wilks [1] with inclusion of mass concentration in the presence of Soret and Dufour along with boundary conditions are given as under.

$$
\begin{aligned}
& \frac{\partial u}{\partial x}+\frac{\partial v}{\partial y}=0 \\
& u \frac{\partial u}{\partial x}+v \frac{\partial u}{\partial y}=v \frac{\partial^{2} u}{\partial y^{2}}-g \beta\left(T-T_{O}\right)-g \beta\left(C-C_{0}\right)-\frac{v}{k} u \\
& u \frac{\partial T}{\partial x}+v \frac{\partial T}{\partial y}=k \frac{\partial^{2} T}{\partial y^{2}}+\frac{D_{m} K_{T}}{c_{s} c_{p}} \frac{\partial^{2} C}{\partial y^{2}} \\
& u \frac{\partial C}{\partial x}+v \frac{\partial C}{\partial y}=D_{B} \frac{\partial^{2} C}{\partial y^{2}}+\frac{D_{m} K_{T}}{T_{m}} \frac{\partial^{2} T}{\partial y^{2}}
\end{aligned}
$$

where $u$ and $v$ are the $x$ and $y$ components of the velocity, gfi( $\mathrm{T}_{\mathrm{i}}$ $\left.\mathrm{T}_{\mathrm{o}}\right)$ is buoyancy force in thermal diffusion, $\mathrm{gfi}\left(\mathrm{C}_{\mathrm{i}} \mathrm{C}_{\mathrm{o}}\right)$ is buoyancy force in chemical diffusion, ${ }^{\circ}$ is the kinematic viscosity, $g$ is the gravitational

where $U$ is the free stream velocity

$u=v=0, T=T_{1}, C=C_{1}$ at $y=0$

$u \rightarrow U, T \rightarrow T_{0}, C \rightarrow C_{0}$ at $y \rightarrow \infty$

\section{Dimensionless Variables}

Further we introduce the following dimensionless variables to transform the dimensioned field equations into

dimensionless form.

$$
\begin{aligned}
& \bar{x}=\frac{x-x_{s}}{l}, \bar{y}=\frac{\mathrm{Re}^{\frac{1}{2}}}{l} y, \bar{u}=\frac{u}{U}, \bar{v}=\frac{\mathrm{Re}^{\frac{1}{2}}}{U} y \\
& \bar{\theta}=\frac{T-T_{0}}{T_{1}-T_{0}}, \bar{\phi}=\frac{C-C_{0}}{C_{1}-C_{0}}
\end{aligned}
$$

By using (6) in dimensioned field equations (1)-(4) we get the following dimensionless boundary layer equations.

$$
\begin{aligned}
& \frac{\partial \bar{u}}{\partial \bar{x}}+\frac{\partial \bar{v}}{\partial \bar{y}}=0 \\
& \bar{u} \frac{\partial \bar{u}}{\partial \bar{x}}+\bar{v} \frac{\partial \bar{u}}{\partial \bar{y}}=v \frac{\partial^{2} \bar{u}}{\partial \bar{y}^{2}}-\lambda_{T} \bar{\theta}-\lambda_{C} \phi-\frac{\bar{u}}{\operatorname{Re} D a} \\
& \bar{u} \frac{\partial \bar{\theta}}{\partial \bar{x}}+\bar{v} \frac{\partial \bar{\theta}}{\partial \bar{y}}=\frac{1}{\operatorname{Pr}} \frac{\partial^{2} \bar{\theta}}{\partial \bar{y}^{2}}+D u \frac{\partial^{2} \bar{\phi}}{\partial \bar{y}^{2}} \\
& \bar{u} \frac{\partial \bar{\phi}}{\partial \bar{x}}+\bar{v} \frac{\partial \phi}{\partial \bar{y}}=\frac{1}{S c} \frac{\partial^{2} \bar{\phi}}{\partial \bar{y}^{2}}+S u \frac{\partial^{2} \bar{\theta}}{\partial \bar{y}^{2}}
\end{aligned}
$$

In above system of equations, we observed some parameters as follows. $G r=\frac{g \beta\left(T-T_{O}\right)}{v^{2}} l^{3}$ is Grashof number, $\operatorname{Re}=\frac{U L}{v}$ is Reynolds number, $\lambda_{T}=\frac{G_{r}}{R_{e}^{2}}$ is mixed convection parameter of temperature, $\lambda_{C}=\frac{G_{r}}{R_{e}^{2}}$ is mixed convection parameter of mass concentration, $\operatorname{Pr}=\frac{v}{\alpha}$ 
is Prandtl number, $S c=\frac{v}{D_{B}}$ is Schmidt number, $D u=\frac{D_{m} K_{T}\left(C_{1}-C_{0}\right)}{v c_{a} c_{p}\left(T_{1}-T_{0}\right)}$ is Dufour number and $S u=\frac{D_{m} K_{T}\left(T_{1}-T_{0}\right)}{v T_{m}\left(C_{1}-C_{0}\right)}$ is Soret number.

The dimensionless boundary conditions are

$$
\begin{aligned}
& \bar{u}=0=\bar{v}, \bar{\theta}=1, \bar{\phi}=1, \\
& \bar{u} \rightarrow 1, \bar{\theta} \rightarrow 0, \bar{\phi} \rightarrow 0
\end{aligned}
$$

\section{Method of solution}

The dimensionless boundary layer partial differential equations (7)-(10) along with boundary conditions (11) are transformed into convenient form of integration. For this, we introduce the following group of Primitive variable formulation as follows:

\section{Group of primitive variables formulation}

$$
\begin{aligned}
& \bar{y}=Y x^{\frac{1}{4}}, \bar{u}=U x^{\frac{1}{2}}, \bar{v}=V x^{\frac{-1}{4}}, \bar{x}=X, \bar{\phi}=\phi, \bar{\theta}=\theta \\
& X \frac{\partial U}{\partial X}-\frac{1}{4} Y \frac{\partial U}{\partial Y}+\frac{1}{2} U+\frac{\partial V}{\partial Y}=0 \\
& X U \frac{\partial U}{\partial X}+\left(V-\frac{1}{4} Y U\right) \frac{\partial U}{\partial Y}+\frac{1}{2} U^{2}=\frac{\partial^{2} U}{\partial Y^{2}}-\lambda_{T} \theta-\lambda_{C} \phi-\frac{X^{\frac{1}{2}} U}{\operatorname{Re} D a} \\
& X U \frac{\partial \theta}{\partial X}+\left(V-\frac{1}{4} Y U\right) \frac{\partial \theta}{\partial Y}=\frac{1}{\operatorname{Pr}} \frac{\partial^{2} \theta}{\partial y^{2}}+D u \frac{\partial^{2} \phi}{\partial y^{2}} \\
& X U \frac{\partial \bar{\phi}}{\partial \bar{x}}+\left(V-\frac{1}{4} Y U\right) \frac{\partial \phi}{\partial y}=\frac{1}{S c} \frac{\partial^{2} \phi}{\partial y^{2}}+S u \frac{\partial^{2} \theta}{\partial y^{2}}
\end{aligned}
$$

The transformed boundary conditions are

$$
\mathrm{U}=0=\mathrm{V}, \theta=1, \phi=1 \text { at } \mathrm{Y}=0 \text {, }
$$

$\mathrm{U} \rightarrow 1, \theta 0, \phi \rightarrow 0$, as $\mathrm{Y} \rightarrow \infty$

\section{Numerical Method}

The governing equations (13)-(16) along with boundary conditions (17) are solved by finite difference method. These equations are descretized by applying backward difference along $\mathrm{x}$ - direction and central difference along y-direction. The discretization procedure is given as follows:

$$
\begin{aligned}
& \frac{\partial U}{\partial X}=\frac{U_{i, j}-U_{i, j-1}}{\Delta X} \\
& \frac{\partial U}{\partial X}=\frac{U_{i+1, j}-U_{i-1, j}}{2 \Delta Y} \\
& \frac{\partial^{2} U}{\partial Y^{2}}=\frac{U_{i+1, j}-2 U_{i, j}+U_{i-1, j}}{\Delta Y^{2}}
\end{aligned}
$$

By substituting (18) in boundary layer equations 13-16 along with boundary conditions (17) we get the system of algebraic equations. Gauss Elimination method is used to solve this system of algebraic equations. The momentum equation takes the form

$$
A_{1} U_{i+1, j}+B_{1} U_{i, j}+C_{1} U_{i-1, j}=D_{1}
$$

where $A_{1}, B_{1}$ and $C_{1}$ are the unknown coefficients of the variables
$U_{i+1 ; j}, U_{i, j}$ and $U_{i, 1 ; j}$ which are given as

$$
\begin{aligned}
& A_{1}=1-\frac{1}{2} \Delta Y\left(V_{i, j}-\frac{Y}{4} U_{i, j}\right) \\
& B_{1}=-2-\frac{\sqrt{X} \Delta Y^{2}}{\operatorname{Re} D a}-\frac{\Delta y^{2}}{2} U_{i, j}-\frac{\Delta Y^{2}}{\Delta X} X U_{i, j} \\
& C_{1}=1+\frac{\Delta Y}{2}\left(V_{i, j}-\frac{Y}{4} U_{i, j}\right) \\
& D_{1}=-\Delta Y^{2}\left(\frac{X}{\Delta X} U_{i, j} U_{i, j-1}-\lambda_{T} \theta_{i, j}-\lambda_{C} \phi_{i, j}\right)
\end{aligned}
$$

Energy equation takes the form

$$
A_{2} \theta_{i+1, j}+B_{2} \theta_{i, j}+C_{2} \theta_{i-1, j}=D_{2}
$$

where $A_{2}, B_{2}$ and $C_{2}$ are the unknown coefficients of the variables $\theta_{i+1 ; j}, \theta_{i, j}$ and $\theta_{i-1 ; j}$ which are given as

$$
\begin{aligned}
& A_{2}=\frac{1}{\operatorname{Pr}}-\frac{1}{2} \Delta Y\left(V_{i, j}-\frac{Y}{4} U_{i, j}\right) \\
& B_{2}=-\frac{2}{\operatorname{Pr}}-\frac{\Delta Y^{2}}{\Delta X} X U_{i, j} \\
& C_{2}=\frac{1}{\operatorname{Pr}}+\frac{\Delta Y}{2}\left(V_{i, j}-\frac{Y}{4} U_{i, j}\right) \\
& D_{2}=\Delta Y^{2}\left(\frac{X}{\Delta X} U_{i, j} \theta_{i, j-1}+\operatorname{Du}\left(\phi_{i+1, j}-2 \phi_{i, j}+\phi_{i-1, j}\right)\right)
\end{aligned}
$$

Mass equation takes the form

$$
A_{3} \phi_{i+1, j}+B_{3} \phi_{i, j}+C_{3} \phi_{i-1, j}=D_{3}
$$

where $A_{3}, B_{3}$ and $C_{3}$ are the unknown coefficients of the variables $\phi_{i+1 ; j} \phi_{i, j}$ and $\phi_{i-1, j}$ which are given as

$$
\begin{aligned}
& A_{3}=\frac{1}{S c}-\frac{1}{2} \Delta Y\left(V_{i, j}-\frac{Y}{4} U_{i, j}\right) \\
& B_{3}=\frac{2}{S c}-\frac{\Delta Y^{2}}{\Delta X} X U_{i, j} \\
& C_{3}=\frac{1}{S c}+\frac{\Delta Y^{2}}{2}\left(V_{i, j}-\frac{Y}{4} U_{i, j}\right) \\
& D_{3}=-\Delta Y^{2}\left(\frac{X}{\Delta X} U_{i, j} \phi_{i, j-1}+\operatorname{Su}\left(\theta_{i+1, j}-2 \theta_{i, j}+\theta_{i-1, j}\right)\right)
\end{aligned}
$$
below

The velocity can be calculated by using continuity equation as given

$V_{i+1, j}=V_{i-1, j}-\frac{2 X \Delta Y}{\Delta X}\left(U_{i, j}-U_{i, j-1}\right)+\frac{Y}{4}\left(U_{i+1, j}-U_{i-1, j}\right)-\Delta Y U_{i, j}$

The discretized boundary conditions are

$$
\begin{aligned}
& \mathrm{U}_{\mathrm{i}, \mathrm{j}}=0=\mathrm{V}_{\mathrm{i}, \mathrm{j}, \mathrm{i}, \mathrm{j}} \theta_{\mathrm{j}}=1=\phi_{\mathrm{i}, \mathrm{j}} \text { at } \mathrm{Y}=0 \\
& \mathrm{U}_{\mathrm{i}, \mathrm{j}} \rightarrow 1, \theta_{\mathrm{i}, \mathrm{j}} \rightarrow 0, \phi_{\mathrm{i}, \mathrm{j}} \rightarrow 0 \text { at } \mathrm{Y} \rightarrow \infty
\end{aligned}
$$

Equations (13)-(16) form a tri-diagonal system of algebraic equations. These equations are solved by Gaussian Elimination Method. Taking step size $\Delta x=0.05$ and $\Delta y=0.02$, computation is started with 
$x=0$ and marches down implicity. Procedure is repeated up to $10^{-5}$ degree of accuracy. The skin friction, rate of heat and mass transfer are calculated by the following expressions as given below

\section{Results and Discussion}

In this section numerical results have been carried out by Finite Difference Method (FDM) along with physical parameters that is buoyancy force parameter or mixed convection parameter for temperature,$\lambda_{T}$ and mass concentration $\lambda_{C}$, Prandtl number $\mathrm{Pr}$, Schmidt number $S c$, Soret number $S u$ and Dufour number $D u$ on velocity, temperature and mass distribution (Figure 1).

\section{Effects of physical parameters on velocity, temperature and mass distribution}

Figure $2 \mathrm{a}-\mathrm{c}$ is demonstrated to describe the behavior of mixed convection parameter, $\lambda_{T}$ on velocity, temperature and mass

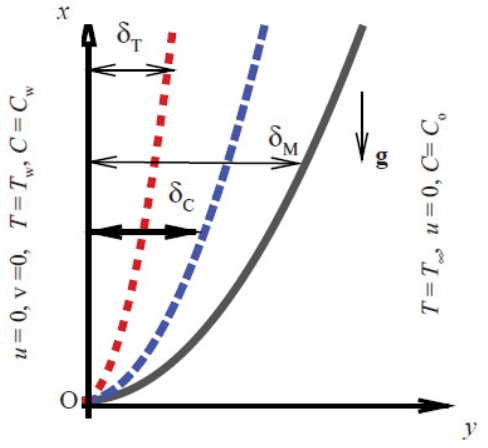

Figure 1: Conguration of ${ }^{\circ}$ ow model. concentration. From these figures it is observed that velocity distribution is an increasing function of mixed convection parameter while temperature and mass are decreasing functions. It is clear that with the increase in buoyancy force parameter, kinetic energy produces which leads to decrease in resistance along the flow, therefore velocity profile increases while temperature and concentration profiles are decreased steadily. The effect of modified mixed convection parameter,$\lambda_{C}$ are shown in Figure $3 \mathrm{a}-\mathrm{c}$. It can be seen that velocity profile is decreased rapidly while temperature and mass profiles are increased gradually with the increase in modified mixed convection parameter. In Figure $4 a-c$, it is concluded that momentum and thermal boundary layer thickness are increased but slight change is noted in concentration boundary layer for various values of Prandtl number Pr. Since Prandtl number is the ratio of kinematic viscosity to thermal diffusivity. Thus for greater value of Prandtl number fluid becomes more viscous and moves slowly due to close interaction molecule form, therefore momentum and thermal boundary layer thickness are increased. For different values of Schmidt number Sc Figure 5a-c portray that velocity is decreasing slightly then attains its asymptotic point and obtained results satisfy boundary condition. It takes place when momentum diffusion dominates over mass diffusion also temperature and mass profiles are decreased slowly and consistently. Figure 6a-c illustrate the inluence of Soret $S u$ number on velocity, temperature and mass concentration profiles. It is seen that velocity profile increases gradually while temperature and concentration profiles decrease slowly and distinctively with the increase of Soret number. This phenomena happens due to large thermal diffusion. The effects of Dufour number on velocity, temperature and concentration profiles are found in Figures $7 \mathrm{a}-\mathrm{c}$. It is clear that velocity and temperature are decreased progressively with the increase in Dufour number $D u$ but there is no change observed in concentration profile.
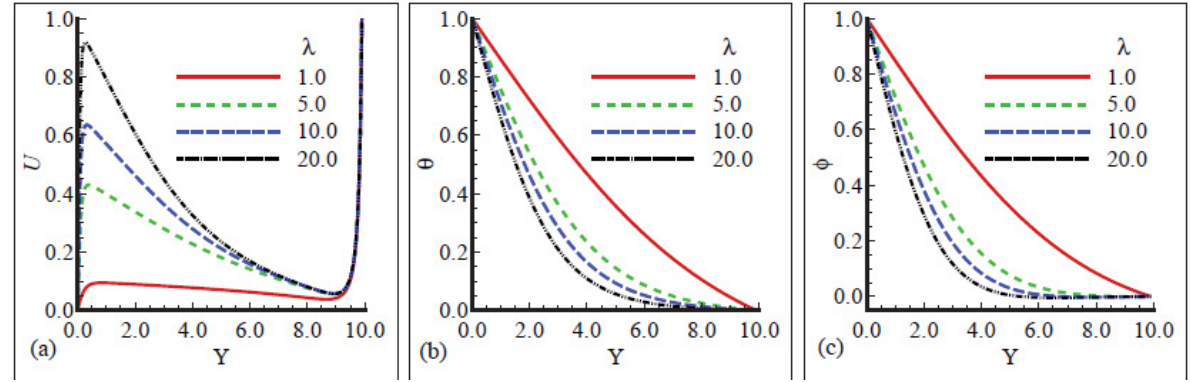

Figure 2: Variation in the (a) velocity distribution (b) temperature distribution (c) mass concentration for di囚erent values of ${ }_{3}$ when $\operatorname{Pr}=0.71, S c=1.0, S u=0.4$, $s_{c}=0.8, D u=0.4$ and $D a=0.5$.
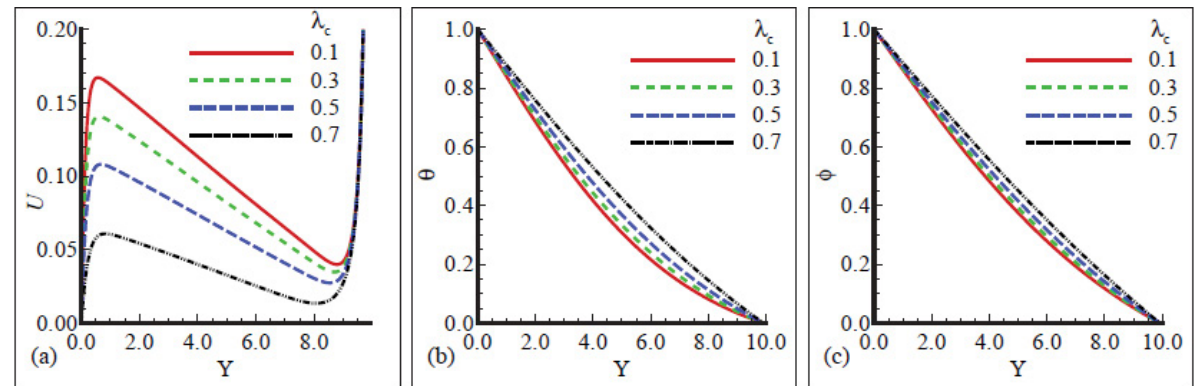

Figure 3: Variation in the (a) velocity distribution (b) temperature distribution (c) mass concentration for dißerent values of ${ }_{s C}$ when $\operatorname{Pr}=1.0, R_{e}=400, S_{c}=0.5$, $S u=0.3, G r=0.8, D u=0.1$ and $D a=0.4$ 
Citation: Ashraf M, Mahmood T, Ahmad U, Hassan W (2018) Soret and Dufour Effects on Heat and Mass Transfer Mixed Convection Flow near a Point of Zero Skin Friction. Fluid Mech Open Acc 5: 184. doi: 10.4172/2476-2296.1000184
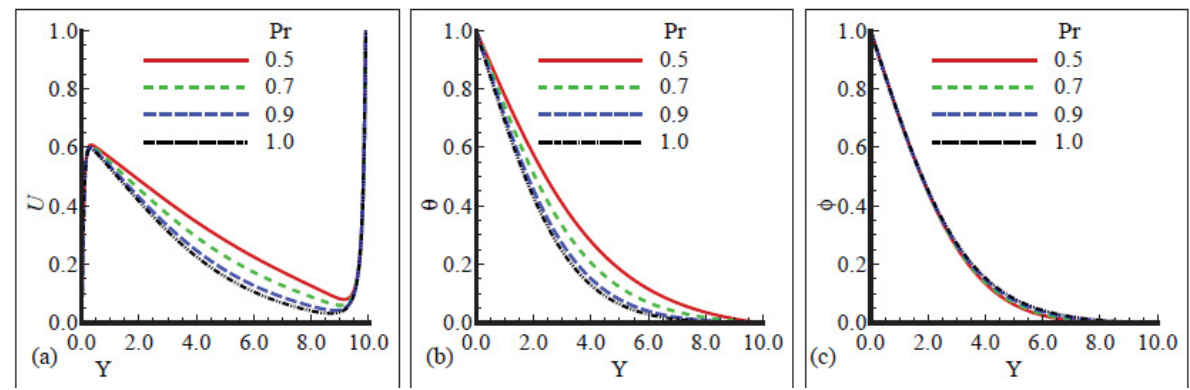

Figure 4: Variation in the (a) velocity distribution (b) temperature distribution (c) mass concentration for dißerent values of $\operatorname{Pr}$ when $,=1.0, s=10.0, R e=$ $400, S c=1.0, S u=0.2, D u=0.3$ and $D a=0.4$.
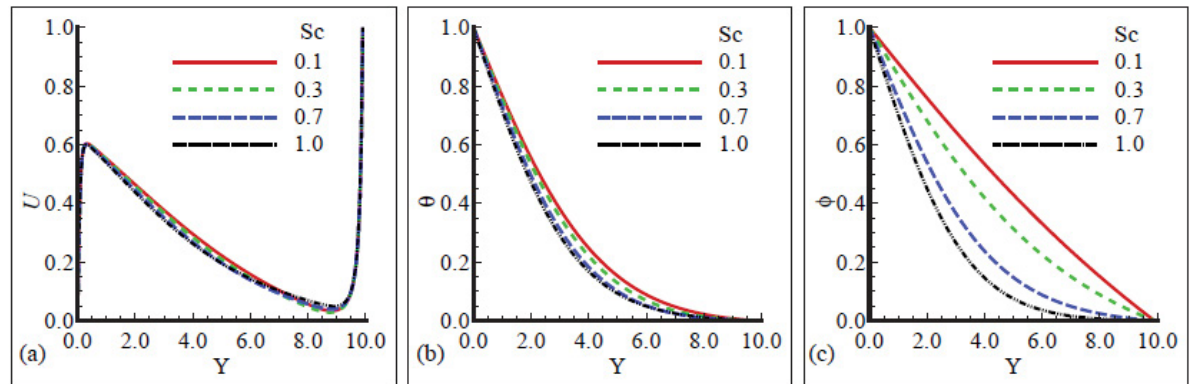

Figure 5: Variation in the (a) velocity distribution (b) temperature distribution (c) mass concentration for dißerent values of $S c$ when $,=1.0, \quad,=10.0, R e=$ $400, \operatorname{Pr}=0.71, S u=0.2, D u=0.5$ and $D a=0.7$
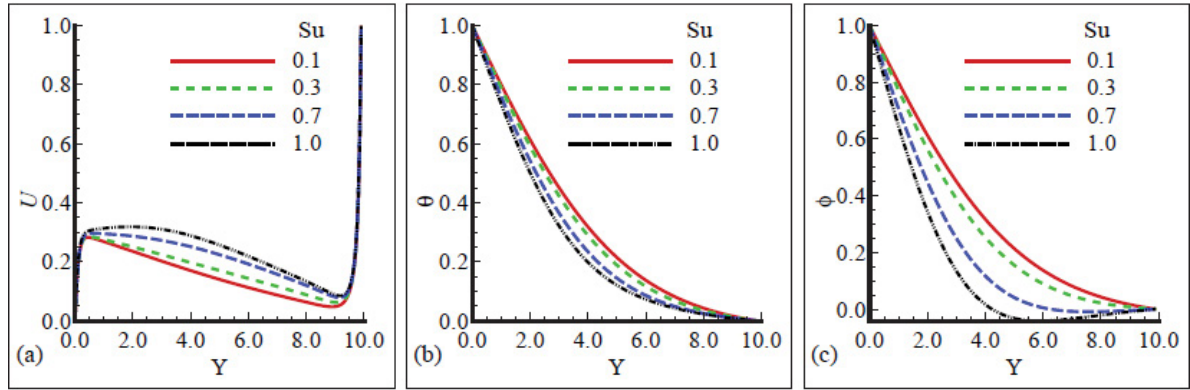

Figure 6: Variation in the (a) velocity distribution (b) temperature distribution (c) mass concentration for different values of $S u$ when $, C=8.0, T=10.0, R e=$ 400, $S c=1.0, \operatorname{Pr}=0.71, D u=0.5$ and $D a=0.7$
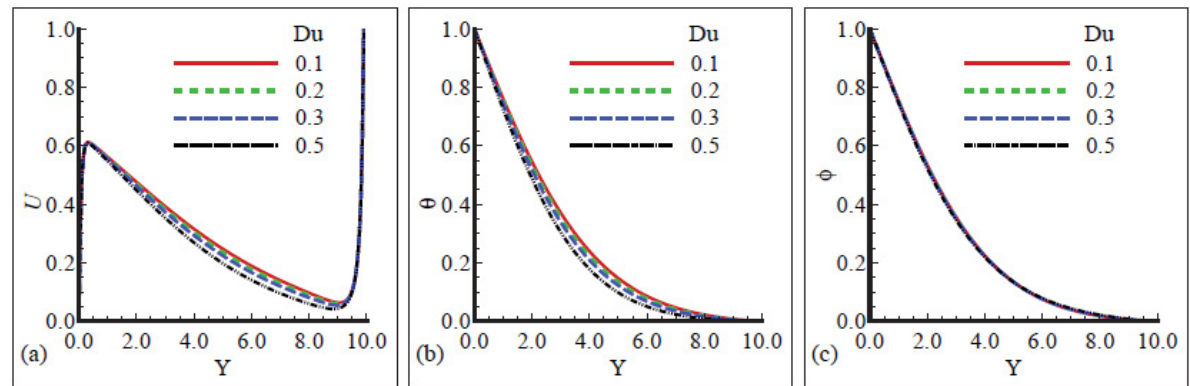

Figure 7: Variation in the (a) velocity distribution (b) temperature distribution (c) mass distribution for dißerent values of $D u$ when $C=0.8, T=10.0, R e=400$, $S c=0.5, S u=0.8, \operatorname{Pr}=0.71$ and $D a=0.3$.

Tables 1-3 illustrate the numerical results of skin friction, rate of heat and mass transfer for different values of mixed convection parameter , $\lambda_{T}$ by keeping other parameters fixed. It is noted that by increasing the value of $X$ skin friction, heat and mass transfer are growing up because mixed convection parameter acts as pressure gradient. The separation point $X_{s}=89$ be the point where the skin friction become uniform or zero having value $Q_{s}=0.30044$ for,$\lambda=1.0$ and $Q_{s}=2.69060$ for,$\lambda=10.0$ at $X_{s}=94$. 
Citation: Ashraf M, Mahmood T, Ahmad U, Hassan W (2018) Soret and Dufour Effects on Heat and Mass Transfer Mixed Convection Flow near a Point of Zero Skin Friction. Fluid Mech Open Acc 5: 184. doi: 10.4172/2476-2296.1000184

Page 6 of 7

\begin{tabular}{|c|c|c|c|}
\hline $\mathbf{X}$ & $\mathbf{T = 1 : 0}$ & $\mathbf{T = 5} \mathbf{0}$ & $\mathbf{T = 1 0 : 0}$ \\
\hline 0.1 & 1.08367 & 5.54992 & 9.6143 \\
\hline 1 & 1.0916 & 5.5918 & 9.68875 \\
\hline 2 & 1.10076 & 5.64016 & 9.77467 \\
\hline 3 & 1.11003 & 5.69058 & 9.86423 \\
\hline 4 & 1.12031 & 5.74321 & 9.9577 \\
\hline 5 & 1.13076 & 5.79824 & 10.05541 \\
\hline 6 & 1.14171 & 5.85589 & 10.15773 \\
\hline 7 & 1.15321 & 5.91637 & 10.26507 \\
\hline 8 & 1.1653 & 5.97997 & 10.3779 \\
\hline 9 & 1.17805 & 6.04697 & 10.49674 \\
\hline 10 & 1.19153 & 6.11774 & 10.62221 \\
\hline
\end{tabular}

Table 1: Numerical values of Skin friction obtained for di®erent values of Mixed convection parameter, $T=1.0,5.0,10.0$, when $P r=0.72, R e=400, S c=1.0, S r=0.8$ $C=0.5, D u=0.5$ and $D a=0.5$

\begin{tabular}{|c|c|c|c|}
\hline $\boldsymbol{X}$ & $\boldsymbol{T = 1 : 0}$ & $\boldsymbol{T = 5 : 0}$ & $\boldsymbol{T = 1 0 : 0}$ \\
\hline 0.1 & 0.13106 & 0.21731 & 0.2609 \\
\hline 1 & 0.13258 & 0.21985 & 0.26395 \\
\hline 2 & 0.13435 & 0.22279 & 0.26749 \\
\hline 3 & 0.13621 & 0.22589 & 0.2712 \\
\hline 4 & 0.13816 & 0.22914 & 0.27511 \\
\hline 5 & 0.14021 & 0.23256 & 0.27922 \\
\hline 6 & 0.14238 & 0.23616 & 0.28355 \\
\hline 7 & 0.14467 & 0.23997 & 0.28813 \\
\hline 8 & 0.14709 & 0.24401 & 0.29297 \\
\hline 9 & 0.14966 & 0.24829 & 0.29812 \\
\hline 10 & 0.1524 & 0.25285 & 0.3036 \\
\hline
\end{tabular}

Table 2: Numerical values of Heat Transfer obtained for di®erent values of Mixed convection parameter, $T=1.0,5.0,10.0$, when $P r=0.72, R e=400, S c=1.0, S r=0.8$, $C=0.5, D u=0.5$ and $D a=0.5$.

\begin{tabular}{|c|c|c|c|}
\hline $\boldsymbol{X}$ & $\boldsymbol{T = 1 : 0}$ & $\boldsymbol{T = 5 : 0}$ & $\boldsymbol{T}=\mathbf{1 0 : 0}$ \\
\hline 0.1 & 0.16646 & 0.28448 & 0.34263 \\
\hline 1 & 0.16843 & 0.28779 & 0.34661 \\
\hline 2 & 0.17072 & 0.29164 & 0.35123 \\
\hline 3 & 0.17311 & 0.29567 & 0.35608 \\
\hline 4 & 0.17563 & 0.2999 & 0.36118 \\
\hline 5 & 0.17827 & 0.30436 & 0.36654 \\
\hline 6 & 0.18106 & 0.30906 & 0.37219 \\
\hline 7 & 0.184 & 0.31402 & 0.37816 \\
\hline 8 & 0.18712 & 0.31927 & 0.38447 \\
\hline 9 & 0.19042 & 0.32484 & 0.39117 \\
\hline 10 & 0.19394 & 0.33077 & 0.3983 \\
\hline
\end{tabular}

Table 3: Numerical values of Mass Flux obtained for di®erent values of Mixed convection parameter, $T=1.0,5.0,10.0$, when $P r=0.72, R e=400, S_{c}=1.0, S r=0.8$, $C=0.5, D u=0.5$ and $D a=0.5$.

Tables 4-6 demonstrate the results for various values of Prandtl number Pr on skin friction, rate of heat and mass transfer by keeping other parameters constant. From these tables it is seen that skin friction, heat and mass transfer increase as $(X)$ increases. Table 7 displays the comparison of the present results is given with the results given in literature and found be in good agreement.

\section{Conclusion}

Present study discuss the effect of physical parameters as Richardson number,$\lambda_{T}$, modified Richardson number , ${ }_{C}$ Schmidt number $S c$, Reynolds number Re, Prandtl number Pr, Soret number $S u$ and Dufour number $D u$ on velocity, temperature and mass distributions, skin

\begin{tabular}{|c|c|c|c|}
\hline $\boldsymbol{X}$ & $\boldsymbol{P r = 0 . 1}$ & $\boldsymbol{P r = 0 . 3}$ & $\boldsymbol{P r}=\mathbf{0 . 5}$ \\
\hline 0.1 & 0.74664 & 0.74436 & 0.74221 \\
\hline 1 & 0.75228 & 0.7499 & 0.74769 \\
\hline 2 & 0.7588 & 0.75631 & 0.75401 \\
\hline 3 & 0.76561 & 0.763 & 0.76062 \\
\hline 4 & 0.77273 & 0.76999 & 0.76751 \\
\hline 5 & 0.78019 & 0.7773 & 0.77474 \\
\hline 6 & 0.78802 & 0.78498 & 0.78231 \\
\hline 7 & 0.79625 & 0.79304 & 0.79026 \\
\hline 8 & 0.80491 & 0.80154 & 0.79906 \\
\hline 9 & 0.81407 & 0.81005 & 0.80745 \\
\hline 10 & 0.82374 & 0.81997 & 0.81679 \\
\hline
\end{tabular}

Table 4: Numerical values of Skin friction obtained for di®erent values of Prandt number $\operatorname{Pr}=0.1,0.3,0.5$, when $\mathrm{Re}=400, \mathrm{Sc}=1.0, \mathrm{Sr}=0.5, \mathrm{~T}=1.0, \mathrm{C}=0.7, \mathrm{Du}=0.5$ and $\mathrm{Da}=0.5$.

\begin{tabular}{|c|c|c|c|}
\hline $\boldsymbol{X}$ & $\boldsymbol{P r = 0 . 1}$ & $\boldsymbol{P r = 0 . 3}$ & $\boldsymbol{P r = 0 . 5}$ \\
\hline 0.1 & 0.0593 & 0.07697 & 0.09337 \\
\hline 1 & 0.05974 & 0.07781 & 0.09445 \\
\hline 2 & 0.06027 & 0.07879 & 0.09571 \\
\hline 3 & 0.06083 & 0.07983 & 0.09703 \\
\hline 4 & 0.06142 & 0.08091 & 0.09842 \\
\hline 5 & 0.06205 & 0.08206 & 0.09988 \\
\hline 6 & 0.06273 & 0.08328 & 0.10143 \\
\hline 7 & 0.06345 & 0.08457 & 0.10306 \\
\hline 8 & 0.06423 & 0.08594 & 0.10479 \\
\hline 9 & 0.06506 & 0.0874 & 0.10663 \\
\hline 10 & 0.06596 & 0.08896 & 0.1086 \\
\hline
\end{tabular}

Table 5: Numerical values of Heat Transfer obtained for di®erent values of PrandtI number $\operatorname{Pr}=0.1,0.3,0.5$, when $R e=400, S c=1.0, S r=0.5, T=1.0, C=0.7, D u=0.5$ and $D a=0.5$.

\begin{tabular}{|c|c|c|c|}
\hline $\boldsymbol{X}$ & $\boldsymbol{P r}=\mathbf{0 . 1}$ & $\boldsymbol{P r = 0 . 3}$ & $\boldsymbol{P r = 0 . 5}$ \\
\hline 0.1 & 0.11256 & 0.11716 & 0.12114 \\
\hline 1 & 0.11404 & 0.11862 & 0.12269 \\
\hline 2 & 0.11575 & 0.12032 & 0.1244 \\
\hline 3 & 0.11754 & 0.12209 & 0.12619 \\
\hline 4 & 0.11943 & 0.12396 & 0.12808 \\
\hline 5 & 0.12141 & 0.12592 & 0.13006 \\
\hline 6 & 0.12349 & 0.12799 & 0.13215 \\
\hline 7 & 0.12569 & 0.13017 & 0.13435 \\
\hline 8 & 0.12802 & 0.13248 & 0.13669 \\
\hline 9 & 0.13048 & 0.13493 & 0.13917 \\
\hline 10 & 0.1331 & 0.13754 & 0.1418 \\
\hline
\end{tabular}

Table 6: Numerical values of Mass ${ }^{\circ} \mathrm{ux}$ obtained for di®erent values of Prandt| number $\operatorname{Pr}=0.1,0.3,0.5$, when $R e=400, S c=1.0, S r=0.5, T=1.0, C=0.7, D u=0.5$ and $D a=0.5$.

\begin{tabular}{|c|c|c|c|c|}
\hline $\boldsymbol{X}$ & $\begin{array}{c}\text { Skin } \\
\text { friction(present) }\end{array}$ & $\begin{array}{c}\text { Heat } \\
\text { Transfer(Present) }\end{array}$ & $\begin{array}{c}\text { Skin friction } \\
\text { [2] }\end{array}$ & $\begin{array}{c}\text { Heat Transfer } \\
\text { [2] }\end{array}$ \\
\hline 0.04 & 1.42724 & 1.60792 & 1.42299 & 1.60189 \\
\hline 0.15 & 0.31808 & 0.639183 & 0.30933 & 0.69036 \\
\hline 0.19 & 0.00781 & 0.38195 & 0.00157 & 0.43477 \\
\hline
\end{tabular}

Table 7: Comparison of numerical results for skin friction and heat transfer by Present author and Wilks [2].

friction, rate of heat and mass transfer have been studied numerically using finite difference method. we conclude the following important outcomes: The velocity profile increases while temperature and mass concentration profiles decrease with the increase of mixed convection parameter,$\lambda_{T}$, whereas opposite effects exist for modified mixed 
Citation: Ashraf M, Mahmood T, Ahmad U, Hassan W (2018) Soret and Dufour Effects on Heat and Mass Transfer Mixed Convection Flow near a Point of Zero Skin Friction. Fluid Mech Open Acc 5: 184. doi: 10.4172/2476-2296.1000184

convection parameter ${ }_{{ }_{C}}$. Both the momentum and thermal boundary layer thickness increase but slight change occurs in mass concentration boundary layer thickness as Prandtl number Pr increases. With the increase of Schmidt number Sc velocity, temperature and mass profiles are decreased. It can be observed that when we increase the values of Soret number $S u$ velocity increases while temperature and mass concentration profiles decrease. For various values of Dufour number $D u$ velocity and temperature distributions are decreased whereas no change in mass concentration is appeared. It is sighted that skin friction, rate of heat and mass transfer are increased for various values of mixed convection parameter, $\lambda_{T}$ as well as Prandtl number Pr.

\section{References}

1. Hunt R, Wilks G (1980) On the behavior of the laminar boundary -layer equations of mixed convection near a point of zero skin friction. J Fluid Mechanics 101: 377-391.

2. Kafoussias NG, Williams EW (1995) Thermal-diffusion and diffusion-thermo effects on mixed free-forced convective and mass transfer boundary layer flow with temperature dependent viscosity. Inter J Eng Sci 33: 1369-1384.

3. Postelnicu A (2004) Inuence of a magnetic eld on heat and mass transfer by natural convection from vertical surfaces in porous media considering Soret and Dufour effects. Inter J Heat and Mass Transfer 47: 1467-1472.

4. Abreu CRA, Alfradique MF, Telles AS (2006) Boundary layer flows with Dufour and Soret effects: I: Forced and natural convection. Chem Eng Sci 61: 42824289 .

5. Chamkha AJ, Ben-Nakhi A (2008) MHD mixed convectionradiation interaction along a permeable surface immersed in a porous medium in the presence of Soret and Dufours effects. Heat and Mass Transfer 44: 845-856.

6. Beg OA, Beg TA, Bakier AY, Prasad VR (2009) Chemically-reacting mixed convective heat and mass transfer along inclined and vertical plates with Soret and Dufour effects: Numerical solutions. Int J Appl Math Mech 5: 39-57.

7. Sallam SN (2010) Thermal-difusion and difusion-thermo effects on a mixed convection heat and mass transfer in a porous medium. J Porous Media 13 331-345.

8. Makinde OD (2011) On MHD mixed convection with Soret and Dufour e®ects past a vertical plate embedded in a porous medium. Latin Am Applied Res 41: 63-68.

9. Pal D, Mondal H (2011) MHD non-Darcian mixed convection heat and mass transfer over a non-linear stretching sheet with SoretDufour effects and chemical reaction. International Communications in Heat and Mass Transfer 38: 463-467.

10. Gundagani M, Seri S, Reddy CK (2012) Soret and dufour effects on unsteady MHD mixed convection flow past a vertical porous plate with thermal radiation. Caspian J Applied Sci Res 1: 1-13.

11. Chamkha AJ, El-Kabeir SMM (2013) Unsteady heat and mass transfer by MHD mixed convection flow over an impulsively stretched vertical surface with chemical reaction and Soret and Dufour effects. Chem Eng Communications 200: $1220-1236$

12. Srinivasacharya D, Upendar M (2013) Soret and dufour effects on MHD mixed convection heat and mass transfer in a micropolar uid. Central Eur J Eng 3 : 679-689.

13. Chamkha AJ, Rashad AM (2014) Unsteady heat and mass transfer by MHD mixed convection flow from a rotating vertical cone with chemical reaction and Soret and Dufour effects. The Canadian J Chem Eng 92: 758-767.

14. Jha BK, Mohammed U (2014) Mixed convection effect on melting from a vertical plate embedded in porous medium with soret and dufour effects. Heat Transfer Asian Res 43: 667-676.

15. Muthuraj R, Srinivas S, Shukla AK, Ramamohan TR (2014) Effects of thermaldiffusion, diffusion-thermo, and space porosity on MHD mixed convective flow of micropolar fluid in a vertical channel with viscous dissipation. Heat Transfer Asian Res 43: 561-576.

16. Arthur ME, Seini IY, Antwi GD (2015) Analytical solution of dufour and soret effects on hydromagnetic flow past a vertical plate embedded in a porous medium. Advances in Physics Theories and Applications 44: 47-71.

17. Srinivasacharya D, Mallikarjuna B, Bhuvanavijaya R (2015) Soret and Dufour effects on mixed convection along a vertical surface in a porous medium with variable properties. Ain Shams Eng J 6: 553-564.

18. Pal D, Mandal G, Vajravalu K (2016) Soret and Dufour effects on MHD convective-radiative heat and mass transfer of nano fluids over a vertical nonlinear stretching/shrinking sheet. Applied Mathematics and Computation 287 184-200.

19. Ojjela O, Kumar NN (2016) Unsteady MHD mixed convective flow of chemically reacting and radiating couple stress fluid in a porous medium between paralle plates with Soret and Dufour effects. Arabian J Science and Eng 41: 1941 1953.

20. Abel MS, Nandeppanavar MM, Kemparaju MC (2016) Mixed convection hea transfer of MHD flow due to permeable sheet: an analytical solution. Advances in Physics Theories and Applications 52: 21-27. 